\title{
Dependence of Laser Ablation Produced Gold Nanoparticles Characteristics on the Fluence of Laser Pulse
}

\author{
Nasim Mirghassemzadeh ${ }^{1 *}$, Majid Ghamkhari ${ }^{2}$ Davoud Dorranian $^{2}$ \\ ${ }^{1}$ Physics Department, Science Faculty, Islamic Azad University, North Tehran Branch, Tehran, Iran; ${ }^{2}$ Laser Lab., Plasma Physics \\ Research Center, Science and Research Branch, Islamic Azad University, Tehran, Iran. \\ Email: ${ }^{*}$ nasim.mirghassemzadeh@gmail.com
}

Received August $16^{\text {th }}, 2013$; revised September $16^{\text {th }}, 2013$; accepted September $23^{\text {rd }}, 2013$

Copyright (C) 2013 Nasim Mirghassemzadeh et al. This is an open access article distributed under the Creative Commons Attribution License, which permits unrestricted use, distribution, and reproduction in any medium, provided the original work is properly cited.

\begin{abstract}
Effect of laser fluence on the characteristics of gold nanoparticles (NPs) produced by laser ablation method is investigated experimentally. Five samples of gold NPs were synthesized by nanosecond pulsed laser ablation of a high purity gold plate in distilled water at five different fluences in the range of $1-3 \mathrm{~J} / \mathrm{cm}^{2}$. Results show that in this experimental condition gold NPs are produced by nucleation of Au atoms in the plasma, generated on the target surface during the ablation process. There is threshold fluence at which absorption of laser energy by water rises significantly. Absorption of the laser energy by water leads to increase oxygen atoms in the ablation medium and formation of gold oxide NPs. By increasing the laser fluence below the threshold, size of produced NPs decreases while by increasing the laser fluence above the threshold size of produced NPs increases.
\end{abstract}

Keywords: Laser Ablation; Nanoparticle; Absorption Spectra; XRD; TEM; SEM; Mie Theory; Surface Plasmon Resonance

\section{Introduction}

There is a growing interest in the fabrication of nanomaterials and their applications in various fields of life and technology such as electronics, health care, energy generation, and storage $[1,2]$. Nanotechnology is the understanding and control of matter at dimensions between approximately 1 and 100 nanometers, where unique phenomena enable novel applications. Encompassing nanoscale science, engineering, and technology, nanotechnology involves imaging, measuring, modeling, and manipulating matter at this length scale. Unusual physical, chemical, and biological properties can emerge in materials at the nanoscale [3].

The convenient methods to produce these particles can be categorized in three chemical, electrical, and laser ablation methods. Evidence shows that the latter method is superior to other methods. Different structural and morphological characters of produced NPs can be controlled by laser fluence, spot size, wavelength, pulse width, and repetition rate of laser pulse. The influence of

${ }^{*}$ Corresponding author. ablation environment on the characters of NPs is negligible. Indeed, laser ablation in liquids, which consists of the pulverization of a solid target in liquid ambience, gives a unique opportunity to solve the toxicity problems. In contrast to chemical nanofabrication methods, laser ablation can be performed in a clean, well-controlled environment, such as deionized water, giving rise to the production of ultrapure nanomaterials. The use of these particles decreases toxicity risks, which is especially important in vivo bio sensing and imaging applications [4-8].

Noble metal NPs have attracted much attention because of their unique size dependent optical properties, magnetic properties, and catalytic properties. The size dependent properties of gold NPs make them suitable for many applications in various areas such as catalytic, optical and antibacterial applications [9-11].

Gold NPs are proved to be more effective as it has good antimicrobial efficacy against bacteria, viruses and other eukaryotic micro-organisms. The current investigations support that use of gold ion or metallic gold as well as gold NPs can be exploited in medicine for burn treat- 
ment, dental materials, coating stainless steel materials, textile fabrics, water treatment, sunscreen lotions, etc. and posses low toxicity to human cells, high thermal stability and low volatility [12-15].

In this manuscript we have investigated the effect of laser fluence on the characteristics of gold NPs produced by laser ablation of a gold bulk in distilled water. Effect of laser pulse energy on the size distribution as well as lattice structure of NPs is studied experimentally and morphology of nanoparticle is investigated.

The manuscript is organized as follow; following the introduction in Section 1, the experimental setup is presented in Section 2. Section 3 is devoted to results and discussion and finally Section 4 includes conclusion.

\section{Experimental Setup}

NPs were prepared by ablation of a high purity gold bulk in distilled water, using the fundamental harmonic of a $\mathrm{Nd}$ :YAG laser operating at $1064 \mathrm{~nm}$ with pulse width of $7 \mathrm{~ns}$ and $10 \mathrm{~Hz}$ repetition rate. Gold bulk was placed at the bottom of a water container with its surface at the focal point of a $80 \mathrm{~mm}$ convex lens. Height of water on the gold target was $12 \mathrm{~mm}$. Laser beam diameter was 6 $\mathrm{mm}$ before lens and is calculated to be $30 \mu \mathrm{m}$ on the surface of the target. The volume of the water in the ablation contain was $20 \mathrm{ml}$ and gold target was ablated with 1000 laser pulses at different energies. Samples 1 - 5 were prepared with laser pulse fluences of 3, 2.5, 2, 1.5, and 1 $\mathrm{J} / \mathrm{cm}^{2}$ respectively, which are introduced in Table 1.

Optical absorption spectra of samples in a $10 \mathrm{~mm}$ path length quartz cells were measured by UV-Vis-NIR spectrophotometer from PG Instruments (T-80). Dynamic Light Scattering (DLS) measurement was done using the Nano ZS (red badge) ZEN 3600 device from Malvern Co, for studying the hydrodynamic size distribution of the NPs in water. TEM and SEM micrographs were taken using EM208 and XL30 systems form PHILIPS Co respectively. X-ray diffraction was measured employing X PERTPRO X-ray diffract-meter from the PANalitical.

To measure the ablation mass rate in the liquid medium, the target was weighed up before and after the ablation process by an accurate Sartorius utensil model CP225D with $0.01 \mathrm{mg}$ readability. After ablation inside water with 1000 laser pulses, the target was dried and weighed up again. Hence, the mass loss of the target attributed to the amount of generated NPs could be measured.

\section{Results and Discussion}

In Figure 1, photo of samples are presented and in Figure 2 the ablation rate of samples versus laser fluence is plotted. The color of sample 1 is dark red which changes gradually to bright red for sample 5. In Figure 2 it is shown that the ablation mass from the target is increased with increasing the laser pulse fluence. In this range of gold nanoparticle production, the ablation rate varies about $5 \times 10^{-7}$ gr to $2 \times 10^{-6}$ gr per pulse. Depending on nanoparticle size and concentration, the color of gold nanoparticle solution in water can be varied. In the case of nanoparticle size less than $100 \mathrm{~nm}$, the gold nanoparticle solution in water is red which changes to dirty yellowish for large size particles. The color of our samples are all red, confirm that the size of produced NPs are in the same range $(\sim 10-50 \mathrm{~nm})$. But their color changes from dark red to bright red. This could be because of concentration of NPs in the solution which is shown in Figure 2. With increasing the laser fluence the ablation rate increases. Increasing the ablation rate versus laser fluence is not linearly and its slope increases in with laser fluence.

The XRD pattern of dried powder of generated NPs on silicon and the X-ray diffraction spectrum of the Au target are shown in Figure 3. All have a poly crystal structure with several preferred orientations. Diffraction peaks are clearly observed and are located in positions consistent with those expected for $\mathrm{Au}$, as indicated by the Joint Committee on Powder Diffraction Standards (JCPDS). The preferred orientation of Au bulk target and generated NP samples are not exactly the same. Various Bragg reflections clearly indicate the presence of $\langle 111\rangle,\langle 200\rangle$, $<220>,<311>$, and $<222>$ sets of lattice planes in the structure of NPs which are exactly the same with the planes in the structure of bulk target. Results show that during the ablation processes gold oxide, $\mathrm{Au}_{2} \mathrm{O}_{3}$, NPs are also produced in the water solution. The peaks at $2 \theta=$ $34^{\circ}$, and $43^{\circ}$, and $52^{\circ}$, respectively corresponds to $<040>$ and $<511>$ and $<222>$ planes of $\mathrm{Au}_{2} \mathrm{O}_{3}$. There were not such Bragg reflection peaks in the spectrum of solid target. Therefore, it can be claimed that in this regime of ablation, $\mathrm{Au}$ atoms bond breaks by laser pulse energy and they recombine again during the cooling process of plasma plume generated on the surface of the target. During the recombination process some gold atoms bond with oxygen atoms in the water and gold oxides molecules are formed. From sample 1 to sample 3, the inten-

Table 1. Laser fluence and the wavelength correspond to SPR of samples.

\begin{tabular}{cccccc}
\hline Sample & 1 & 2 & 3 & 4 & 5 \\
\hline Laser fluence $\left(\mathrm{J} / \mathrm{cm}^{2}\right)$ & 3 & 2.5 & 2 & 1.5 & 1 \\
SPR wavelength $(\mathrm{nm})$ & $522-524$ & $520-524$ & $516-520$ & $518-522$ & $524-526$ \\
Hydrodynamic size $(\mathrm{nm})$ & 32 & 32 & 42 & 42 & 52 \\
\hline
\end{tabular}




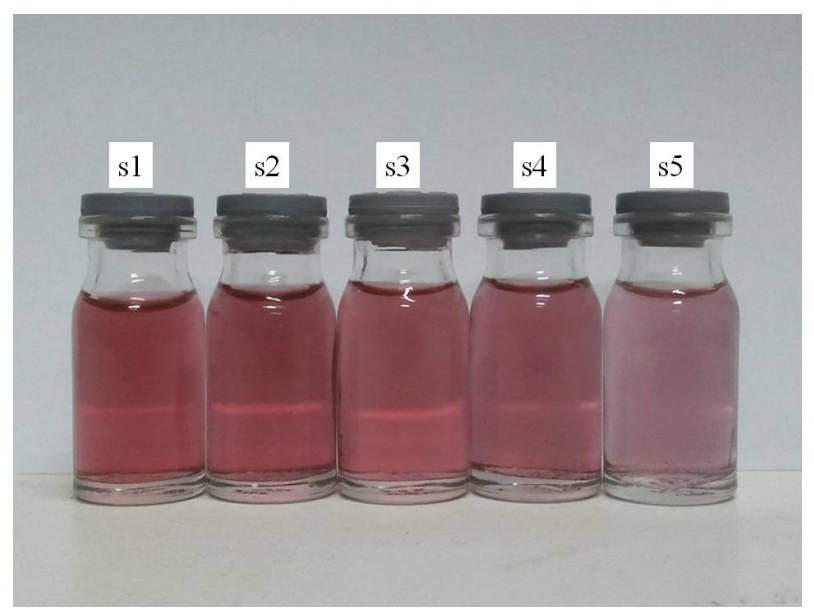

Figure 1. Au NPs in distilled water prepared by laser ablation process. Darker color is due to higher number of nanoparticles in the suspensions because of higher laser fluence during the ablation process.

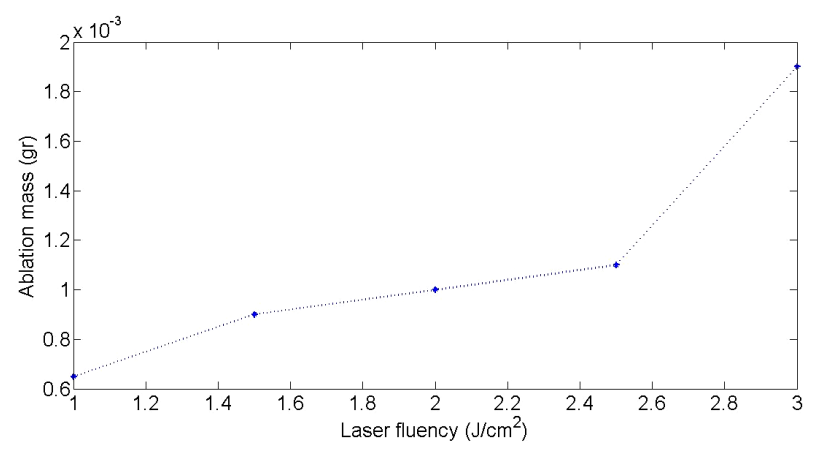

Figure 2. Variation of the mass of the target during the ablation versus laser pulse fluence for $\mathbf{1 0 0 0}$ laser pulses.

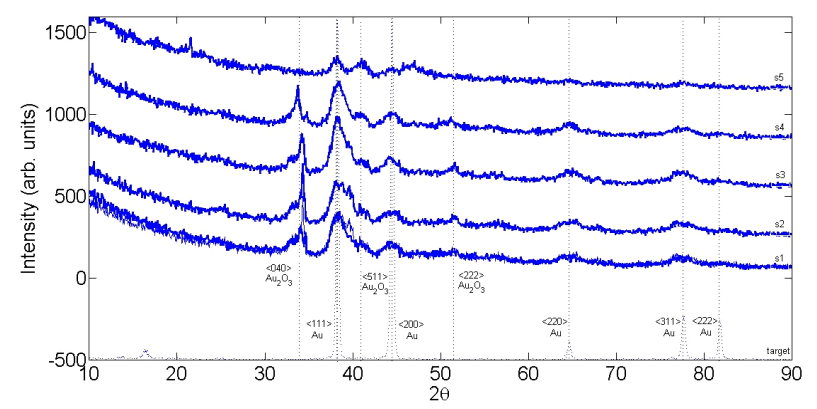

Figure 3. X-ray diffraction pattern of Au target and Au nanoparticle after drying the suspensions on silicon at room temperature.

sity of peaks of XRD pattern (correspond to both $\mathrm{Au}$ and $\mathrm{Au}_{2} \mathrm{O}_{3}$ ) is increased while from sample 3 to sample 5 the intensity of peaks are decreased, which is may be due to the number of NPs in samples.

Additionally, the mean grain size of synthesized $\mathrm{Au}$ NPs could be estimated according to the Debye-Scherrer equation. Broadening the peaks of XRD pattern of NPs in comparison with bulk peaks confirm that size of grains is decreased in the generated NPs in comparison with bulk Au. Among the XRD patterns of different samples, peaks of s3 are broadest. It can be due to the difference in the size of grains because of different laser pulse energy in the ablation process, show that grain size of NPs in s3 is smaller than the grain size of NPs in other samples.

The noticeable point is the absence of gold oxide in $\mathrm{s} 5$, where the laser fluency was $1 \mathrm{~J} / \mathrm{cm}^{2}$ (minimum). So in the process of nanoparticle production in water, there is threshold laser fluence at which formation of gold oxide NPs in water starts.

Absorption spectrums of nanoparticle solutions are presented in Figure 4. The absorption peak is occurred at about $516-526 \mathrm{~nm}$ for all samples due to Au NPs Surface Plasmon Resonances (SPR). For SPR phenomenon to happen, the particle must be much smaller than the wavelength of incident light [9]. If the size of NPs increases, their SPR absorption peak will be shifted toward larger wavelength and vise-versa. It can be seen in Figure 4 that the intensity of absorption peaks are different. The intensity of absorption peak depends on the number of NPs in the suspensions. For different samples this number is different in the experiment. From sample 1 to sample 5, with decreasing the laser fluence, the intensity of SPR peaks is decreased. This result is natural and in a very good agreement with the result of Figure 2, which confirm that from sample 1 to 5 the number of Au NPs in the suspensions is decreased. The wavelength corresponds to SPR peaks are written in Table 1. From sample 1 to 3 a blue shift of few nanometers is occurred for the wavelengths corresponds to peaks but from sample 3 to 5 this shift is occurred toward the large wavelength. These shifts are due to particle size in suspensions,shows that from s1 to $\mathrm{s} 3$ with increasing the laser fluence the size of nanoparticles in suspensions is decreased while from s3 to s5 with decreasing the laser fluence size of produced nanoparticles is increased.

Morphology of gold NPs is studied using the SEM micrographs which are presented in Figure 5. In these pictures suspensions were dried on the aluminum foil. Pictures were taken using $25 \mathrm{keV}$ electrons leads to 7.5

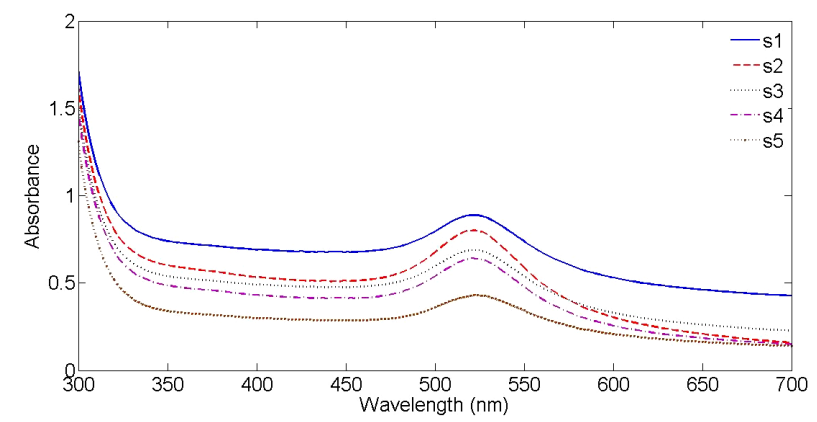

Figure 4. UV-Vis-NIR absorption spectrum of Au NPs in distilled water, with distilled water as the reference. 


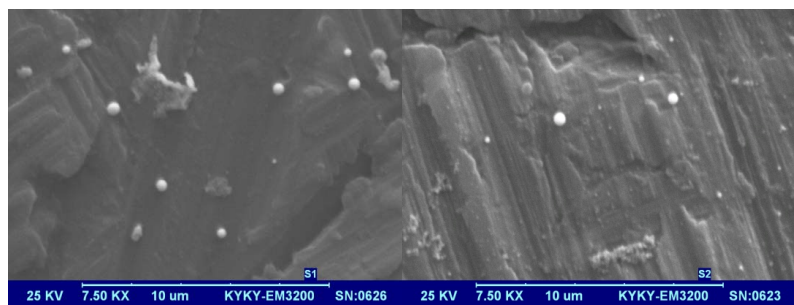

$\mathrm{S} 1$

$\mathrm{S} 2$

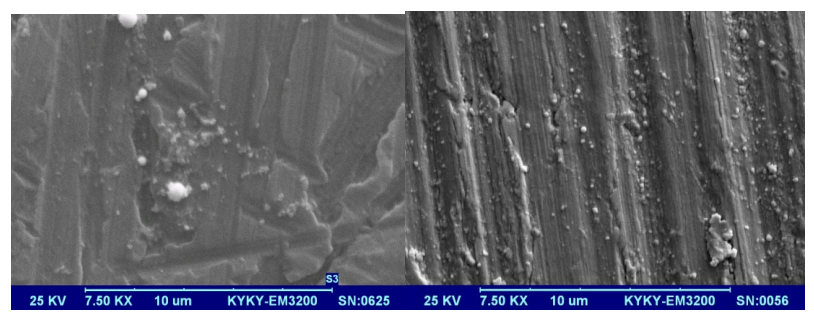

S3

S4

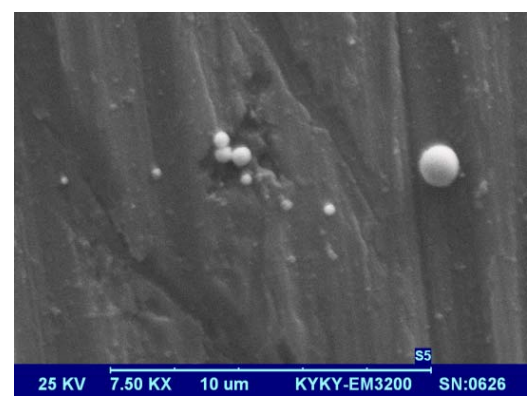

S5

Figure 5. SEM micrographs of Au nanoparticle samples dried on aluminum foil.

$\mathrm{k} \times$ magnification. The morphology of nanoparticles is strongly depends on the energy and wavelength of laser pulse in the experiment as well as the ablation environment [4]. Particles prepared by laser pulse in this experimental condition are spherical nanostructures. The amount of NPs is increased by increasing the laser pulse energy. In this regime of ablation process NPs are separate without any noticeable adhesion. Again in this result the effect of laser pulse energy on the size of NPs can be observed. From s1 to s3, with increasing the laser pulse energy number of small particles in the samples is increased, while from sample 3 to 5 more large NPs in the samples can be observed. These different morphologies are strongly depends on the characteristics of generated plasma on the surface of target according to interaction of high power laser pulse with solid target and after that with the plasma itself.

The hydrodynamic radius distribution of particles in suspensions was measured by DLS. Hydrodynamic size of nanoparticles is their real diameter plus the diameter of the electrostatic potential around them, so it is larger than the real size of NPs. The plots of hydrodynamic size distribution of $\mathrm{Au}$ nanoparticles obtained through DLS are presented in Figure 6. For each sample two peaks have been occurred in the size distribution function, show that each suspension contains two classes of NPs with different sizes. Larger NPs in each suspension are produced during the interaction of laser pulse with $\mathrm{Au}$ target at the first step of ablation. Smaller NPs are generated during the interaction of laser pulse with the produced NPs in the first step of ablation leads to fragment them. The area under each peak of curves is proportional to the number of particle of that size. In this case the sizes of majority of NPs in the suspensions are distributed around the larger size number. The peak of the larger size of NPs in each sample is written in Table 1. Considering the peaks correspond to larger NPs in the suspensions, hydrodynamic size of nanoparticles is decreased from sample 1 to 2 and is increased from sample 2 to 5 , which is in agreement with other results. There is not any noticeable order for the size peaks of smaller NPs in suspensions. They all show NPs of 12 to $18 \mathrm{~nm}$ in the samples due to the further illumination of ablated NPs.

TEM images of Au nanoparticles are presented in Figure 7. For doing TEM measurement a drop of the suspension containing nanoparticles is deposited and dried on a carbon-coated copper grid. Beside each image in Figure 7, the particle size distribution of gold nanoparticles, obtained from TEM analysis is plotted. As can be seen, the gold nanoparticles are almost spherical in all samples and the particle sizes and size distribution are related to the laser pulse energy. The images indicate that with increasing the laser fluence the average size of the spherical nanoparticles decreases from sample 1 to 3 , and then with further increasing the laser fluence from sample 3 to 5 size of produced NPs is increased. This is in agreement with the observation made in the red and blue shifts of SPR peaks.

\section{Conclusions}

Effect of laser fluence on the characters of gold nanoparticles produced by laser ablation process is studied experimentally. Results confirm that laser fluence is a

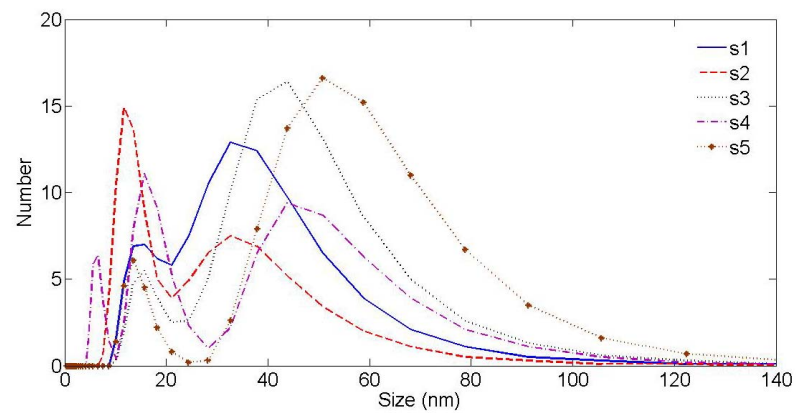

Figure 6. Au nanoparticle hydrodynamic size distribution measured by Dynamic Light Scattering (DLS) device. 

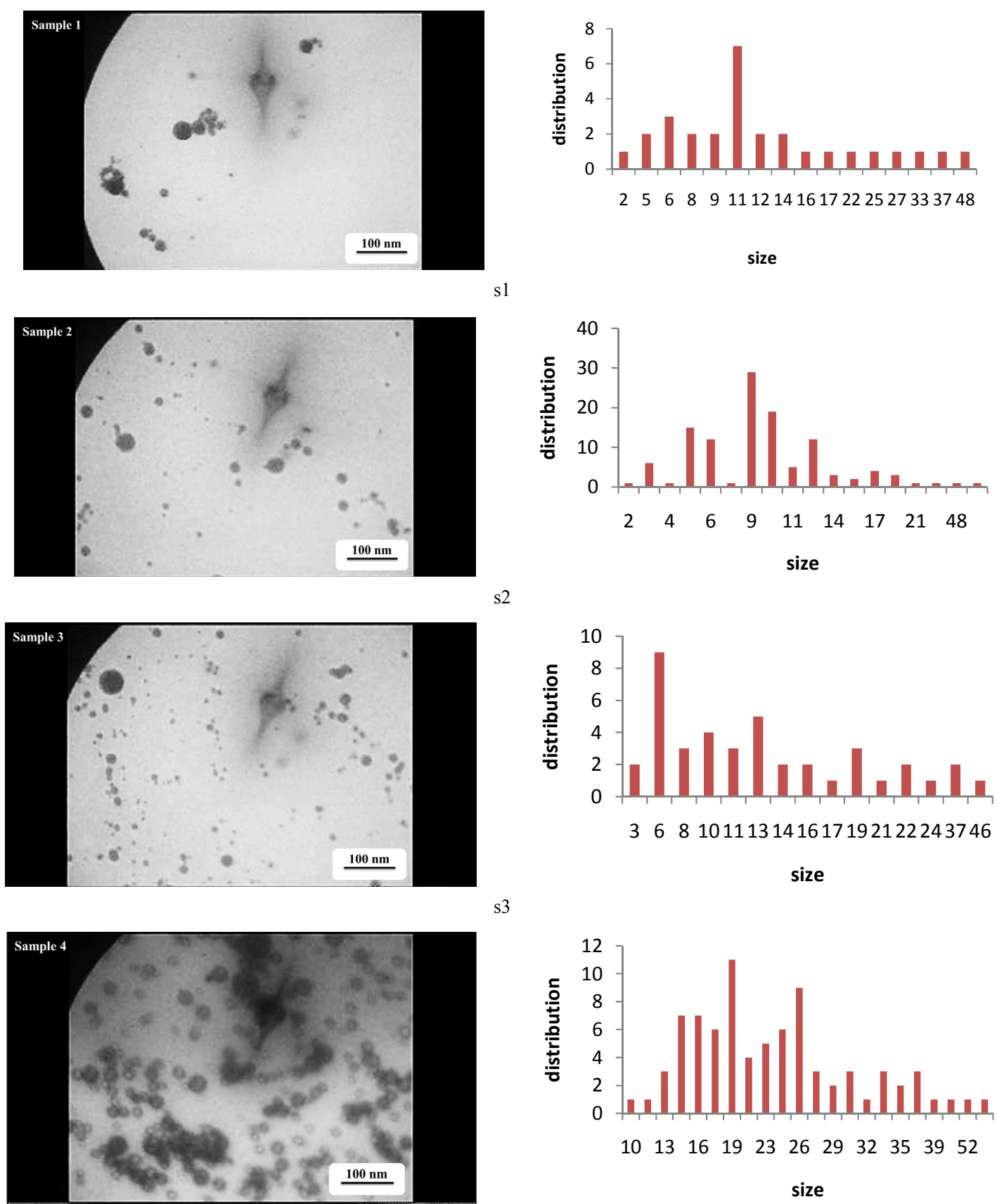

s4
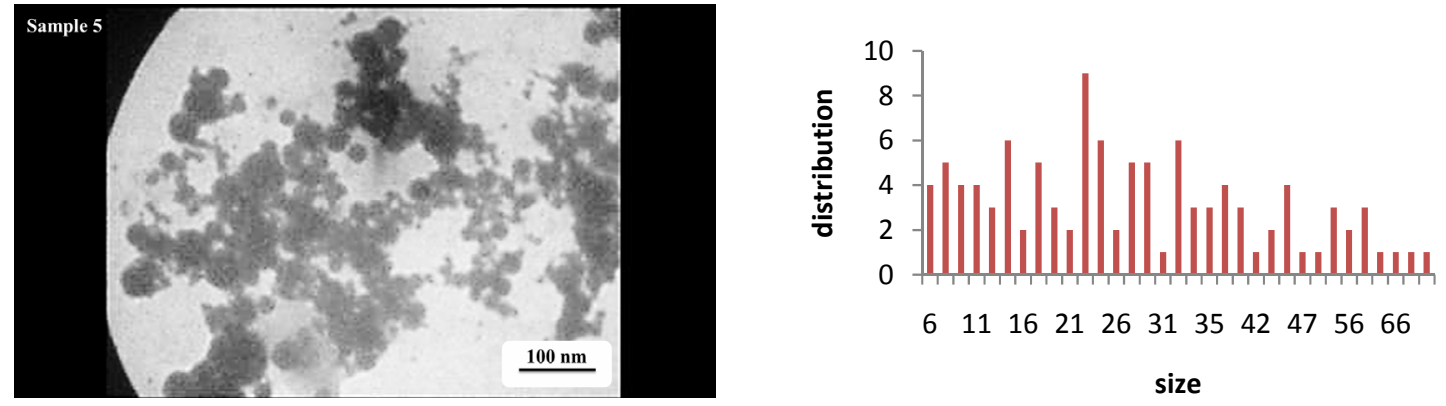

Figure 7. TEM image and size distribution of Au nanoparticle generated in distilled water with laser ablation mechanism. 
powerful tool to control the morphology and size of NPs. Size and morphology of nanoparticles produced by laser ablation of the solid target in liquid medium depends on the magnitude of laser energy which touches the target surface through liquid medium. Results show that by increasing the laser fluence in this experimental condition the size of produced gold nanoparticles will decreased. But there is a limit for increasing the laser fluence. Our investigation shows that there is threshold for the laser fluence at which the absorption of the laser energy by the liquid environment enhances sharply. Increasing the laser pulse energy from this threshold would not lead to decreasing the produced nanoparticles because the large amount of laser pulse energy will be absorbed in liquid environment.

In this experimental condition produced nanoparticles are formed from the nucleation of ablated gold atoms so the lattice structure of nanoparticles is independent of the lattice structure of target.

\section{REFERENCES}

[1] K. Sugioka and M. Meunier, "Laser Precision Microfabrication," 2nd Edition, Cambridge Press, London 2002.

[2] J. Perriere and E. Millon, "Recent Advances in Laser Processing of Materials," 1st Edition, Elsevier, 2006.

[3] A. Dutt Singh, IJAEST, Vol. 8, No. 1, 2011, pp. 54-57.

[4] D. Dorranian, E. Solati and L. Dejam, "Photoluminescence of ZnO Nanoparticles Generated by Laser Ablation in Deionized Water," Applied Physics A, Vol. 109, No. 2, 2012, pp. 307-314. http://dx.doi.org/10.1007/s00339-012-7073-5

[5] E. Solati, M. Mashayekh and D. Dorranian, "Effects of Laser Pulse Wavelength and Laser Fluence on the Characteristics of Silver Nanoparticle Generated by Laser Ablation," Applied Physics A, Vol. 112, No. 3, 2013, pp. 689-694. http://dx.doi.org/10.1007/s00339-013-7812-2

[6] D. Dorranian, S. Tajmir and F. Khazanehfar, Accepted to publish in Soft Nano Letters.
[7] I. Anghela, F. Jipaa, A. Andreia, S. Simiona, R. Dabua, A. Rizeab and M. Zamfirescua, "Femtosecond Laser Ablation of $\mathrm{TiO}_{2}$ Films for Two-Dimensional Photonic Crystals," Optics \& Laser Technology, Vol. 52, 2013, pp. 6569. http://dx.doi.org/10.1016/j.optlastec.2013.04.020

[8] J. P. B. Silva, K. C. Sekhar, A. Almeida, J. Agostinho Moreira, M. Pereira and M. J. M. Gomes, "Influence of Laser Repetition Rate on Ferroelectric Properties of Pulsed Laser Deposited $\mathrm{BaTiO}_{3}$ Films on Platinized Silicon Substrate," Applied Physics A, 2013.

[9] M. Rashidian and D. Dorranian, "Effect of Concentration on the Plasmonic Absorption and Optical Nonlinearity of Gold Nanoparticles," Optical Engineering, Vol. 51, No. 8, 2012, Article ID: 089001. http://dx.doi.org/10.1117/1.OE.51.8.089001

[10] V. Amendola, S. Polizzi and M. Meneghetti, "Laser Ablation Synthesis of Gold Nanoparticles in Organic Solvents," The Journal of Physical Chemistry B, Vol. 110, No. 14, 2006, pp. 7232-7237. http://dx.doi.org/10.1021/jp0605092

[11] I. D. Mayergoyz, D. R. Fredkin and Z. Zhang, "Electrostatic (Plasmon) Resonances in Nanoparticles," Physical Review B, Vol. 72, No. 15, 2005, Article ID: 155412. http://dx.doi.org/10.1103/PhysRevB.72.155412

[12] P. Chandra, D. Das and A. A. Abdel Wahab, Digest Journal of Nanomaterials and Biostructures, Vol. 5, No. 2, 2010, pp. 363-367.

[13] M. Wang and M. Thanou, "Targeting Nanoparticles to Cancer," Pharmacological Research, Vol. 62, No. 2, 2010, pp. 90-99. http://dx.doi.org/10.1016/j.phrs.2010.03.005

[14] J. L. Li, L. Wang, X. Y. Liu, Z. P. Zhang, H. Ch. Guo, W. M. Liu and S. H. Tang, "In Vitro Cancer Cell Imaging and Therapy Using Transferrin-Conjugated Gold Nanoparticles," Cancer Letters, Vol. 274, No. 2, 2009, pp. 319-326. http://dx.doi.org/10.1016/j.canlet.2008.09.024

[15] P. Ghosh, G. Han, M. De, Ch. K. Kim and V. M. Rotello, "Gold Nanoparticles in Delivery Applications," Advanced Drug Delivery Reviews, Vol. 60, No. 11, 2008, pp. 1307-1315. http://dx.doi.org/10.1016/j.addr.2008.03.016 